\title{
The Geometry of Basket Weaving
}

\author{
Researchers teamed up with an artist to tweak a popular basket-weaving \\ approach, finding a way to weave ribbons to produce any curvature \\ desired.
}

By Erika K. Carlson $\mathrm{n}$ traditional basket making, one common approach entails weaving ribbons together into a tridirectional array, called triaxial weaving. But the usual way of implementing this method can only produce a limited number of basket geometries. Changyeob Baek, then at the Massachusetts Institute of Technology and the Swiss Federal Institute of Technology Lausanne, and colleagues have shown that using curved ribbons in place of the straight ones typically used in this weaving pattern allows for a much broader range of curvatures and woven shapes [1]. Because the triaxial weave pattern also occurs in contexts such as molecular lattices, understanding the possible geometries of this pattern could lead to discoveries in fields as varied as biology, chemistry, and materials science.

In triaxial weaving, ribbons are laced together to create arrays of hexagons. But a regular array of hexagons, when made from straight ribbons, produces only a flat plane. To introduce curvature, basket makers place topological defects into their

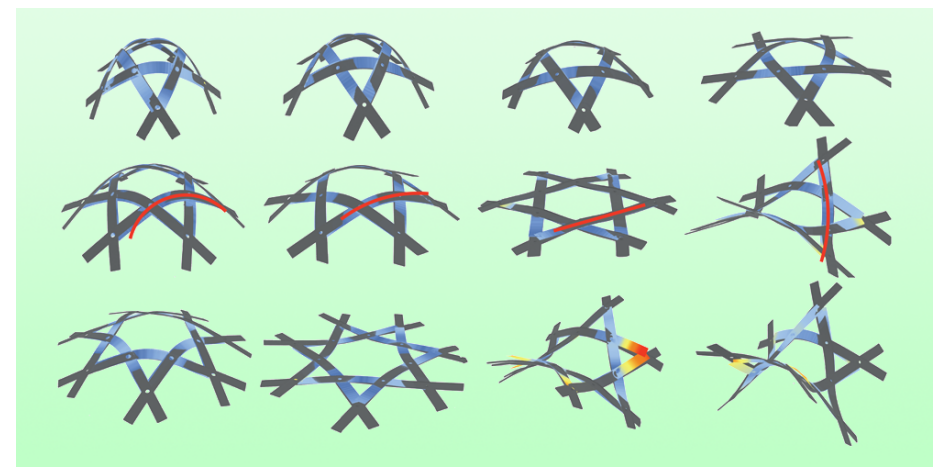

Credit: C. Baek et al. [1] arrays by swapping a hexagon with a pentagon or heptagon. However, these defects can only produce certain discrete degrees of curvature in the straight-ribboned weaves, allowing a limited range of shapes.

Working with an artist who specializes in woven creations, Baek and colleagues investigated a variety of structures woven from ribbons with in-plane curvature. From experiments and simulations, they found that tuning the curvature of the ribbons allowed them to achieve a continuous range of woven curvatures, producing shapes not possible with the traditional straight-ribboned approach. The team also found that different combinations of ribbon geometries and layouts can produce the same shapes; the next step, they say, is to investigate which combinations are the optimal ways to weave specific shapes.

Erika K. Carlson is a Corresponding Editor for Physics based in New York City.

\section{REFERENCES}

1. C. Baek et al., "Smooth triaxial weaving with naturally curved ribbons," Phys. Rev. Lett. 127, 104301 (2021). 